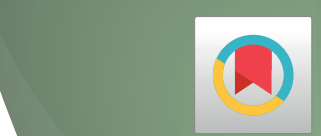

\title{
La mediación en los proyectos pedagógicos: artistas-docentes como creadores y creadoras de relación desde las artes visuales
}

The Mediation in Pedagogical Projects:

Artists-Teachers as Creators of

Relationship Through the Visual Arts

De mediação em projetos pedagógicos: Artistas-professores como criadores de relações das artes visuais

Rosario García-Huidobro Munita* iD orcid.org/0000-0002-1401-9437 Catalina Montenegro González ** iD orcid.org/0000-0002-4432-5582

Para citar este artículo: García-Huidobro, R. y Montenegro-González, C. (2021). La mediación en los proyectos pedagógicos: artistas-docentes como creadores y creadoras de relación desde las artes visuales. Revista Colombiana de Educación, /(82), 83-106. https://doi.org/10.17227/rce.num82-10587

\begin{tabular}{lr}
\hline (c) $\bigcup_{\text {BY }} \$$ & Recibido: $04 / 10 / 2019$ \\
Evaluado: $14 / 08 / 2020$
\end{tabular}

* Doctora en Artes y Educación, Universidad de Barcelona. Docente e investigadora en la Universidad de los Lagos de Chile y directora de la Dirección de Igualdad de Género. Correo electrónico: rosario.garcia-huidobro@ulagos.cl.

** Doctora en Artes y Educación, Universidad de Barcelona. Docente e investigadora en la Universidad de los Lagos de Chile y coordinadora de la mención Artes Visuales de la Carrera Pedagogía en Artes de la universidad. Correo electrónico: catalina.montenegro@ulagos.cl. 


\section{Resumen}

Este artículo, enmarcado en un proyecto de investigación financiado por Fondecyt, sobre las creaciones de los y las artistas-docentes en Chile, busca reflexionar acerca del nuevo hacer de los y las artistas educadoras como quienes promueven la mediación a través de sus propuestas de proyectos artísticos-pedagógicos. Para ello se sitúa a la mediación como un juego de relaciones que se da en el proceso de creación artística y que cuestiona la autoría de la obra, ya que promueve aspectos más vinculados a la escucha y las relaciones reflexivas entre los-as sujetos. Estas ideas se ejemplifican a través del proyecto "La cartografía de los aprendizajes amorosos: reconocimiento de autoridad femenina en los procesos de aprendizaje", desarrollado en el 2018 por una artista-docente en una escuela de Santiago de Chile. A través de la descripción y reflexión del proyecto se señalan cuatro ideas y aportes claves sobre estos tipos de proyectos. Primero es que muestran a la mediación como un lugar para el intercambio y el aprendizaje. Segundo, como una acción que es onto-epistemológica de los y las artistas-docentes contemporáneos. Tercero, la mediación se establece como una característica que los y las sitúa como los y las principales agentes del cambio social desde las artes. Por último, la mediación en estos proyectos se sitúa como una acción política que promueve la subjetividad del estudiantado.

\section{Palabras clave}

Arte; expresión artística; educación artística; mediación entre pares; docentes de artes

\section{Keywords}

Art; art expression; art education; peer mediation; art teacher

\begin{abstract}
This article, framed in a Fondecyt research project on the creations of artists and teachers in Chile, seeks to reflect on the new work of artists-teachers as those who promote mediation through their proposals for artistic-pedagogical projects. For this, mediation is situated as a game of relationships that occurs in the process of artistic creation and questions the authorship of the work, since it promotes aspects more related to listening and reflective relationships between the subjects. These ideas are exemplified through the project "Cartography of love learning: recognition of female authority in learning processes", developed in 2018 by an artist-teacher in a school in Santiago, Chile. Through the description and reflection of the project four ideas and key contributions on these types of projects are indicated. First, they show mediation as a place for exchange and learning. Second, as an action that is onto-epistemological of contemporary artists and teachers. Third, mediation is established as a characteristic that those places as the main transformers of social change from the arts. Finally, mediation in these projects is situated as a political action that promotes the subjectivity of students.
\end{abstract}

\section{Resumo}

Este artigo, baseado em um projeto de pesquisa financiado pelo Fondecyt, sobre as criações de artistas e professores no Chile, busca refletir sobre o novo trabalho de educar artistas como aqueles que promovem a mediação através de suas propostas de projetos artístico-pedagógicos. Para isso, a mediação situa-se como um jogo de relações que ocorre no processo de criação artística e questiona a autoria da obra, pois promove aspectos mais relacionados à escuta e às relações reflexivas entre os sujeitos. Essas ideias são exemplificadas pelo projeto "La cartografía de los aprendizajes amorosos: reconocimiento de autoridad femenina en los procesos de aprendizaje", desenvolvido em 2018 por um artista-professor em uma escola em Santiago, Chile. Através da descrição e reflexão do projeto, são indicadas quatro ideias e contribuições importantes para esses tipos de projetos. Primeiro, eles mostram a mediação como um lugar de troca e aprendizado. Segundo, como uma ação epistemológica de artistas e professores contemporâneos Terceiro, a mediação é estabelecida como uma característica que os coloca como os principais transformadores da mudança social a partir das artes. Finalmente, a mediação nesses projetos situa-se como uma ação políicica que promove a subjetividade do corpo discente.

\section{Palavras-chave}

Arte; expressão artística educação artística; mediação de pares; professores de artes 


\section{Introducción}

Este artículo se sitúa en el marco del primer año del proyecto Fondecyt 11180057, titulado "Nuevos vínculos entre lo artístico y lo pedagógico: Abriendo campos hacia una reconceptualización del arte y el rol del-la artista en la sociedad actual". Este proyecto, desarrollado en Chile, tiene por objetivo conocer y comprender cómo los y las artistas visuales que se desempeñan como docentes (en educación formal o informal) vinculan la práctica artística con la pedagógica y cómo estos cruces tienen el potencial de crear nuevas propuestas en la producción artística y la educación artística en Chile. Para lograr este objetivo nos hemos propuesto cuatro objetivos más específicos, dos de los cuales se exploran en este artículo. El primero, describir las relaciones, los desafíos y las potencialidades que se originan entre los procesos de creación artística y las experiencias pedagógicas de los y las artistas visuales que enseñan. El segundo, describir los elementos que caracterizan los proyectos artísticos que desarrollan los y las artistas-docentes y analizar cuáles son sus aportes al área artística de nuestro país.

Nos centraremos en los objetivos mencionados, dado que en el primer año de este proyecto se ha realizado una indagación exploratoria que ha permitido identificar, registrar y visibilizar a diversos artistas-docentes en Chile, quienes desarrollan proyectos que vinculan lo artístico con lo pedagógico. Estas propuestas dan cuenta de los procesos reflexivos en torno a cuáles son los cruces entre las artes y la educación y cuáles son los desafíos que estos tipos de proyectos plantean cuando se piensan el rol social de las artes y la educación artística. En este proceso de revisión de proyectos artísticos-pedagógicos se evidenció un concepto relevante para esta investigación: la mediación. Esta revisión nos llevó a pensar en las artes visuales y sus prácticas como actos mediadores que promueven el cambio y la transformación social (Barbosa, 2002; Moreno, 2016; Rodrigo y Collados, 2015), ya que se transforman en prácticas que abren y desarrollan nuevas subjetividades. De aquí que en este escrito buscaremos responder la pregunta acerca de si la educación y los espacios artísticos en contextos educativos son lugar de la mediación.

En relación a las propuestas revisadas durante esta primera fase del estudio, valoramos el desarrollo de algunos proyectos artísticos pedagógicos que han ido incorporando una serie de nuevos elementos, como la mediación. Antes, el tipo de arte aislado, individual o de taller no consideraba elementos sociales y relacionales, los que hoy se transforman en el eje de acción de un arte que afecta, lo que trae otros significados al campo del arte y nos permite ampliar los cuestionamientos sobre su rol en la sociedad (García-Huidobro, 2019, 2020). 
Frente a ello, la figura del docente y la docente de artes o artista educador/educadora se vuelven cruciales para este estudio, ya que el posicionamiento onto-epistemológico desde donde se sitúa considera que el trabajo y desarrollo profesional de estos agentes contemporáneos es un saber situado y variado, que contiene en sí mismo una acción pedagógica. Comprendemos que dichos saberes son múltiples y responden a la multiplicidad de funciones o roles que desempeñan en su día a día (ser profesor o profesora, artista, aprendiz, mediador o mediadora). Estas ideas guardan relación con cuestiones ontológicas, ya que apostamos por artistas-docentes que no se piensan de una manera rígida o cerrada, sino múltiple, híbrida, flexible en la que concilian varias formas de ir siendo (García-Huidobro, 2018a; 2018b; Hall, 2010).

A partir de las consideraciones anteriores, en este estudio se supone que el sentido pedagógico va más allá de los espacios formales o informales de enseñanza, y se sitúa, más bien, en todos los aprendizajes de la vida cotidiana en donde damos sentido social a la experiencia (Lucke, 1999; Zúñiga, 2019). Asimismo, no se piensa a la práctica artística y cultural como un objeto que se hace o produce, si no como un encuentro para hacer y pensar (De Pascual y Lanau, 2018). Desde este lugar, entonces, destacamos que la práctica artística y cultural de los y las artistas-docentes es pedagógica porque, como expresa el colectivo Nubol, en su proceso existe sorpresa, aprendizaje y acciones transformativas. A esto se suman los planteamientos de Joseph Beys y su modelo de pedagogía como arte (Cercós y Raichs, 2017), y la dificultad de separar las artes de la enseñanza. Desde estas premisas, un proceso creativo será pedagógico al permitirnos aprender y repensar las subjetividades. Este proyecto de investigación da por hecho que la subjetividad de los y las artistas visuales contemporáneos que enseñan se posiciona desde un cruce compartido entre el sentido de ser artista y docente. También, se cree que el quehacer del y la artista-docente enriquece nociones tradicionales sobre la identidad del y la artista y abre nuevas ideas y visiones sobre la creación artística, donde observamos que emergen el compromiso político, la creatividad y la transformación social (Ossa, 2017).

Las ideas mencionadas han situado este estudio en una metodología cualitativa, emergente y flexible. Esto ha sido crucial en el desarrollo de esta investigación, ya que la metodología no ha sido considerada como un hacer fijo y lineal, sino como una apertura y posibilidad de serpenteo, o como expresaría Pathi Lather (2013), sobre dejarnos perder en la investigación. Conocer los cruces entre lo artístico y lo pedagógico requiere analizar elementos onto-epistemológicos de quienes se viven como artistas-docentes, lo que es un recorrido plural, polifónico, de múltiples capas y sentidos. En este estudio, esta multidimensionalidad ha sido indagada cruzando herramientas de diferentes metodologías en sus etapas. 
La primera fase, de la cual emerge este artículo, en su inicio solo estuvo orientada a una revisión bibliográfica y de literatura sobre los conceptos de práctica artística, sentido pedagógico, mediación, lo colectivo, afectivo, el cambio o transformación social desde las artes, entre otros. Sin embargo, las distintas voces y discusiones bibliográficas sobre estos conceptos nos llevaron a buscar algunos casos de artistas y artistas-docentes y conocer con mayor profundidad sus proyectos, para comprender cómo estos elementos revisados emergían en el cruce de sus propuestas. Para ello, se buscó a artistas, artistas-docentes y colectivos tanto en Chile como en otras partes del mundo, que realizaran proyectos donde combinaran lo artístico con lo pedagógico para promover nuevos significados de las artes visuales y subjetividades. Se contactó a tres artistas-docentes chilenas, una mexicana y otra de Nueva York.

Las artistas-docentes chilenas relataron proyectos artísticos desarroIlados en contextos educativos de escuelas públicas en el país. La artista mexicana relató el proyecto artístico SPIA (Calderón y Ortiz, 2019), desarroIlado en un contexto universitario, y la artista estadounidense compartió el proyecto artístico UnTeaching, desarrollado en una galería de Nueva York en el 2019. También participaron dos colectivos de artes y educación. EI primero fue el grupo de artistas-docentes chileno NubeLab, que relataron el proyecto expositivo "Tú, yo y las cosas de este lugar", desarrollado en Sala de Arte ccu en el 2019. Por último, participó el colectivo de artistas-docentes llamado Pedagogías Invisibles, con el relato de la exposición y programa de actividades "Ni arte ni educación", desarrollado en el Centro de Creación Contemporánea Matadero Madrid en el 2013.

Al comprender que sus proyectos artísticos y pedagógicos eran posibilitadores de nuevas subjetividades en las artes visuales, se les invitó a que escribieran sobre sus procesos para dar cuenta de los cruces entre las artes y la educación (García-Huidobro, 2020). Se decidió no entrevistarlos, sino dar lugar a sus escritos como fuentes de saber. Para ello, se les pidió que escribieran sobre sus proyectos artísticos y pedagógicos y que los contaran y describieran. Sus escritos deberían incluir una reflexión acerca del sentido de este cruce y cómo percibían que sus proyectos impactaban en las áreas de las artes y la educación. La invitación a relatar sus procesos fue muy importante, ya que se consideraron elementos de la investigación narrativa, en la cual sus relatos escritos se transformaban en una manera de aprender y vía de conocimiento social y personal (Chase, 2011). En la primera etapa de esta investigación, estos escritos dieron sentido y encarnaron la revisión bibliográfica, por eso no fueron codificados.

En la segunda fase de este proyecto de investigación, se ha trabajado con diversos grupos en Chile (artistas, mediadores y mediadoras, artistas-docentes, estudiantes de licenciatura en Artes y estudiantes de Pedagogía en Artes). A través del método de Talleres Artísticos de Discusión (García-Huidobro,

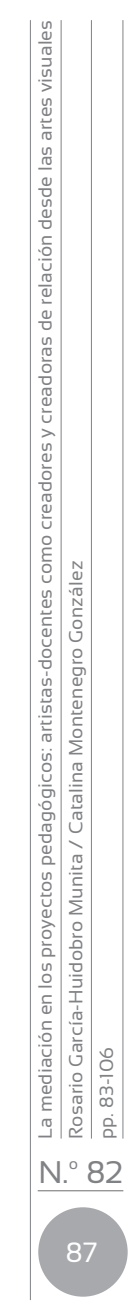


s. f.), se les ha invitado a trazar los cruces entre lo artístico y lo pedagógico en sus experiencias y a reflexionar acerca de sus aportes sociales. El trabajo con estos grupos no perdió de vista lo aprendido con los casos revisados en la primera fase.

Los hallazgos más relevantes de la primera etapa de investigación, que aluden a la revisión bibliográfica y a los casos revisados, nos permiten reflexionar sobre cómo las artes se han ido re-conceptualizando a través de la labor pedagógica, una que incorpora elementos de la mediación, lo colectivo, afectivo y el cambio o transformación social como estrategias que diversos artistas educadores y educadoras han utilizado. Esto ha permitido abrir, en el campo de las artes, el lugar de la relación como una nueva forma de entender, cuestionar y expresar lo artístico (García-Huidobro, 2017).

Para explicar cómo hemos dilucidado algunos cruces entre lo artístico y lo pedagógico en este estudio, el artículo se centra en reflexionar sobre la mediación, como un elemento distintivo que emergió en alguno de los casos de estudio explorados en la primera fase. Para ello, se describirá el proyecto de una de las artistas-docentes chilenas que compartió sus experiencias y se reflexionará sobre cómo emergen los elementos mediadores en la propuesta. El proyecto artístico-pedagógico que se presenta fue titulado "La cartografía de los aprendizajes amorosos: reconocimiento de autoridad femenina en los procesos de aprendizaje". Este proceso se desarrolló en el Liceo n. ${ }^{\circ} 1$ de niñas Javiera Carrera, en Santiago de Chile en el 2018 y fue promovido por una artista-docente que se desempeñaba como profesora de Artes Visuales en dicha escuela. Consideramos que este proyecto expone elementos relevantes sobre los nuevos desafíos de los y las artistas-docentes y nos permite reflexionar en torno a los aportes que están realizando para resignificar el campo de las artes visuales y el rol de los y las artistas como mediadores y mediadoras de la vida social y cultural.

Para ello, en los siguientes apartados ahondaremos en explicar parte de la revisión bibliográfica que se analizó durante la primera fase de este estudio. Esta estuvo vinculada al giro que han realizado las artes gracias al repensar educativo y cómo ha surgido la noción de mediación en las propias prácticas artísticas, como una forma de integrar nuevas relaciones y experiencias con lo artístico. También indagaremos en la mediación como una estrategia política en las artes y como una característica de los y las artistas docentes que buscan generar transformaciones en diversos contextos. Luego encarnaremos dichas conceptualizaciones, describiendo y reflexionando en torno al trabajo creativo y pedagógico del proyecto "La cartografía de los aprendizajes amorosos: reconocimiento de autoridad femenina en los procesos de aprendizaje", el cual nos permitirá repensar el lugar de la mediación y la relación en los proyectos artísticos educativos para mostrar nuevos significados de las artes visuales en la sociedad actual. 


\title{
Cuando las artes visuales se abren y se reposicionan desde lo social
}

Cuando tomamos la creación artística como punto de partida de posibles reflexiones, podemos tomar ciertas posturas, según seamos docentes, artistas o mediadores y mediadoras culturales. Frente a esto, nuestro postulado es que no importa desde qué posición iniciemos el recorrido, sino cómo pensemos, experimentemos, materialicemos y practiquemos las artes visuales, pues dicho posicionamiento onto-epistemológico es una invitación a no entendernos linealmente, más bien como un cruce de experiencias que nos re-sitúan. Tomamos la noción de posicionamiento como una forma de construir subjetividad, pues ambos son:

\begin{abstract}
Procesos por el cual las personas se construyen conjuntamente dentro de determinados contextos sociales y culturales, con el fin de encontrar un lugar dentro de ellos, permitiendo una diversidad de posicionamientos e identidades dentro de dichas interacciones discursivas con los demás. (Carraco y García-Huidobro, 2017, p. 65)
\end{abstract}

En este sentido, desde el lugar donde nos observamos y miramos a las artes, podemos hacernos diversas preguntas, como por ejemplo, si considerar a las artes visuales como un medio para algo o un fin en sí mismo. Al respecto, Brown nos ha expresado que "en un mundo donde hacer preguntas reflexivas y pensar críticamente parece un mínimo histórico, quizás el arte debería hacer mucho más" (s. f., p. 1). Si consideramos la invitación de W. Keith Brown, ¿hacia dónde apuntarían esas reflexiones? ¿Cuál sería el rol que cumpliría el arte como mediación, creación o formación? ¿Es el arte el lugar de la reflexión? ¿Cómo el arte puede promover nuevas relaciones y formas de entendernos?

En la sociedad contemporánea, las artes visuales nos invitan a reflexionar sobre la propia forma de hacer sociedad, sobre las creaciones de protesta y contemplación, sobre el arte como fin, como una herramienta para poner en diálogo y como un lugar de incomodidad y extrañeza que nos permite cuestionar(nos). Independientemente de ello, consideramos que lo importante es comprender y reflexionar sobre cuál es su rol en la sociedad en la que existimos. Aquí cumple un rol fundamental la figura de artistas como creadores y creadoras, pero también como mediadores y mediadoras culturales pedagógicos, para dar sentido social a las artes visuales.

Trabajar con personas requiere de habilidades sensibles, mucha escucha, reflexión y autoestudio sobre cuestiones de raza, clase, género, sexualidad, privilegios, capitalismo y economía política. Trabajando en las comunidades son una gran responsabilidad y los artistas que hacen este trabajo deben ser responsabilizados de maneras que no creo 
que el mundo del arte haya tenido que considerar antes. Trabajar con otros lleva tiempo, paciencia, consulta, confianza de la comunidad, etc. (Brown, s. f., p. 2)

De esta manera, las prácticas socio-artísticas cobran un rol fundamental en un momento de nuestra historia, donde la creación artística es también mediación y práctica social. Desde estas ideas podemos considerar las artes visuales como un proceso mediador y de transformación para abordar problemáticas sociales, proponiendo una práctica artística y cultural que propende por establecer relaciones entre el campo de la cultura y las posibles acciones sociales, donde las derivas, los procesos y las experiencias no tendrán necesariamente una relación con obras como producto artístico. Esto se plantea, entonces, como una valoración de las relaciones entre personas y los procesos de creación artística como un encuentro reflexivo que permite abordar las complejidades sociales mediadas por las propias artes visuales.

Estas ideas han sido reforzadas por Irit Rogoff (2011), quien ha señalado que se puede observar un giro educativo en las prácticas artísticas y comisariales-curatoriales, ya que ha entrado una perspectiva más pedagógica. Aunque Rogoff advierte que este giro aún es confuso, es necesario comprender que las exposiciones ya no se consideran como simples montajes de obras, ni la educación como una transmisión ni adquisición de conocimientos y, por lo tanto, las relaciones entre las prácticas artísticas y la educación serían mucho más complejas de descifrar desde la perspectiva curatorial, lo que necesita la escuela para vincularse con el arte y viceversa. De esta manera, se podría pensar que "el giro educativo en las prácticas curatoriales funciona principalmente como un giro exclusivo de los comisarios, que instrumentalizan la educación en sus propios intereses, constatándose así el inmovilismo en la relación de poderes de las prácticas expositivas" (Sternfeld, 2010, citado en Coca, 2011, p. 72).

Por tanto, es legítimo preguntarse: ¿Es genuino el interés del arte y sus prácticas curatoriales por relacionarse con los espacios de aprendizaje? ¿Se necesitan mutuamente? Es posible que estas preguntas no sean explícitas en los equipos educativos de los espacios culturales o de las escuelas, pero lo cierto es que han adquirido cada vez más importancia las prácticas artísticas con sentido educativo y de mediación y transformación social, lo que ha llevado a vincular las escuelas con los centros culturales no solo por la propia inquietud de hacerlo, sino también porque hay cada vez más programas, tanto de instituciones privadas como gubernamentales, que potencian, propician y financian estas relaciones.

Este proceso de transformación no implica que los educadores deban convertirse en comisarios, sino que el objetivo es que estos profesionales pedagógicos puedan participar en los procesos de conceptualización dis- 
cursiva del museo y apostar por la creación de narrativas que miren por los intereses del público, implementando metodologías de interpretación y construcción de un conocimiento. Fomentar nuevas actitudes en los visitantes significa diseñar estrategias que permitan atender a sus características impulsando acciones que generen experiencias significativas. (Coca, 2011, p. 73)

En este sentido, se plantea al (la) artista como agente de cambio que no solo puede crear, sino que, por sobre todo, media y acompaña un proceso de cambio, respecto a las necesidades de un contexto. Es aquí cuando el trabajo de un artista visual se vuelve fundamental, puesto que su hacer ya no está únicamente vinculado con el trabajo plástico individual y su reconocimiento no está dado por la autoría de la obra (Hernández, 2011). Hablamos, entonces, de artistas que en su acción artística incluyen la pedagogía como ingrediente primordial, porque se transforma para ellos y ellas en un espacio para aprender, sorprenderse y disfrutar. Esta idea nos lleva a pensar en la mediación no solo como un lugar para el intercambio, sino más bien como una acción onto-epistemológica de los y las artistas-docentes contemporáneos y una característica que los y las sitúa como los y las principales agentes del cambio social desde las artes.

\section{El lugar de la mediación como propiciadora de crítica, cambio social y apuesta para las artes}

¿Podemos pensar en la figura de artista como
un agente mediador? Según Marcel Duchamp
el artista es en sí mediador.

Duchamp, The creative act

Es posible comprender la mediación como una dinámica dialógica crítica que permite poner en tensión las artes visuales y los contextos sociales y, por tanto, plantea que el artista o la artista es necesariamente un(a) agente de crítica social y no disociado(a) de esta última. Así, podemos pensar en las artes visuales y sus prácticas como actos mediadores para la transformación social, comprendidas como la confluencia de los procesos artísticos, la sociedad y la cultura, como lo expresa Moreno: "creación artística como un elemento generador de pensamiento y como un espacio de libertad del individuo" (2016, p. 35), además de una práctica social que refleja la cultura y posibilita "la emergencia de diversas prácticas que no se alejen del momento histórico en el que se producen" (Ramos, 2019, p. 80) y, así, establecer una producción del conocimiento desde la propia colectividad. En la misma línea, se releva el rol mediador de las artes visuales como un elemento de transformación social (Barbosa, 2002; 
Moreno, 2016; Rodrigo y Collados, 2015), que da importancia al proceso de la creación y al carácter colaborativo de las experiencias y valora las colectividades, estableciendo relaciones de confianza entre pares y permitiendo un desarrollo artístico basado en la práctica dialógica.

A nivel histórico, la mediación comenzó su desarrollo durante la segunda mitad del siglo xx, a través de las comunidades artísticas del Reino Unido y Norteamérica, donde artistas se involucraban en diversos proyectos a través de instituciones culturales. De esta manera, el acto de mediar de los y las artistas se planteó también como una forma de plantear y proponer posicionamientos en la sociedad que se habitaba. Estas ideas nos invitan a pensar, entonces, que la creación artística se articula como obra y mediación "y es en este sentido que podemos plantear, también, el objeto artístico como un agente de mediación, el cual contiene, por sí mismo, una agencia en lo que se refiere a tejer y deshacer las redes" (Fontdevila, 2018, p. 46), dando énfasis a los procesos y a la comprensión de la obra también como un ente mediador.

Desde estas ideas, podríamos pensar en dos formas de mediar, una relativa al acto del (la) artista/mediador-mediadora/educador-educadora en su proceso de creación y otra, de la obra como tal. Así, la obra trascendería en el tiempo y sería constante en su mediación. Visto así, el acto creativo es también un acto dialógico crítico, donde convergen las discusiones que afectan también a la sociedad.

En este sentido, se pueden nombrar las iniciativas de artistas como Joseph Beuys, Judy Chicago y Miriam Schapiro como la conciencia de la mediación a través de la creación artística en la década de los setenta, pioneros y pioneras de estas ideas que, en el caso de Chicago y Schapiro, desafían "constantemente el mundo del arte dominado por los hombres" (http://www.artnet.com/artists/judy-chicago/) buscando también Ilamar la atención con elementos tradicionalmente descartados por el arte, como los elementos cerámicos y la costura de telas. De esta manera, los espacios artísticos también se transforman en lugares autogestionados que ponen en valor las actividades cotidianas de colectivos cuyo lugar no estaba puesto en los espacios tradicionales artísticos. Ejemplo de esto es la obra The dinner party de Judy Chicago, donde prepara una mesa con telas bordadas y platos de cerámica creados para invitar a la mesa a mujeres que fueron parte de la historia universal. Otro ejemplo de suma relevancia son las arpilleras bordadas de Violeta Parra, donde pone en valor el relato de las dinámicas sociales excluidas, fuera del contexto de creación artística tradicional y toma elementos materiales como telas de bajo costo (sacos y arpilleras) para ser trabajados con lanas de colores, donde hace dialogar temáticas religiosas, indígenas y de la tradición rural chilena, transformándose en un "reflejo de la complejidad mestiza del imaginario popular" (Hormazabal, 2013, p. 6). 
Rogoff (2008), por su parte, plantea que la tendencia de las artes visuales hacia la educación es una forma de identificar cuando una cosa importante está siendo dicha y el mediador o la mediadora adquieren relevancia. Según esto, la propia mediación sería permanecer en un no lugar, en la grieta desde donde se desplaza hacia otras posibilidades de legitimarse, pero en el caso de la educación formal ¿No sería ese lugar, la grieta desde donde llevamos a cabo una reflexión en torno a las artes visuales que no se apropian de la autoría ni se visibiliza a través de la creación artística? Entonces, ¿la mediación sería un lugar más discreto dentro de los circuitos de creación artística? ¿Es la educación ese lugar discreto de la mediación?

En un contexto educativo existen múltiples posibilidades de posicionamiento, lo que nos permite circular por los diversos roles y prácticas artísticas, llevando a cabo procesos reflexivos que muchas veces no son clasificables, más discretos o sin legitimidad autoral, lo que no los hace menos importantes. Aun cuando podemos pensar en la no autoría, existe un proceso reflexivo que permea las diversidades de la escuela y la cultura, y permite transitar de la no autoría a la autoría colectiva, mediante dinámicas constantes de reflexión sobre las artes, sus preocupaciones y los procesos mediadores transformativos. Se podría plantear que la mediación es un proceso rizomático, desjerarquizado y ausente de ego, además de ser un propiciador de la escucha y las relaciones reflexivas.

Según este planteamiento, las artes visuales podrían tener una influencia social tan potente que el artista o la artista actúan como agentes mediadores en los contextos sociales, llegando incluso a proponer nuevas miradas desde las artes visuales. Su influencia puede ser tal, que Oscar Wilde la ejemplifica en su libro La decadencia de la mentira (1898) como la idea de una naturaleza que imita al arte, y J. M. W. Turner afirma que son los atardeceres imperfectos y deslavados los que quieren parecerse a una pintura atmosférica.

¿Poseen, entonces, las artes una responsabilidad como mediadoras

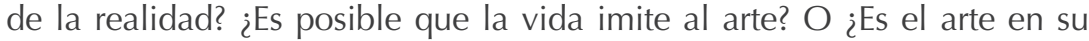
relación con la realidad la que se va mimetizando con lo cotidiano? Quizás es relevante plantear la necesidad de una relación estrecha de las artes visuales con la vida en un rol mediador y, por tanto, serán también las manifestaciones artísticas las encargadas de rescatar las manifestaciones de esa vida, la sociedad y la cultura.

\section{Un relato pedagógico que promueve la mediación y se resignifica desde la relación y las artes}

Con el fin de reflexionar en torno a la importancia de la mediación como un elemento transversal que emergió en algunos proyectos artísticos pedagógicos y que posibilita un lugar de cambio para las artes y la 
valoración sociocultural de los procesos en contextos educativos, en este apartado relataremos uno de los proyectos revisados en la primera fase de este estudio.

El proyecto artístico pedagógico que se presenta, se titula "La cartografía de los aprendizajes amorosos: reconocimiento de autoridad femenina en los procesos de aprendizaje" y se desarrolló en el Liceo n. ${ }^{\circ} 1$ Javiera Carrera de Santiago. Este proceso estuvo a cargo de una artista educadora del Liceo, en un curso de segundo año medio (estudiantes mujeres de entre 15 y 16 años), durante el segundo semestre del 2018.

El proyecto emergió de la necesidad de la artista-docente y de las inquietudes de las estudiantes por visibilizar el rol de las mujeres en los procesos de aprendizaje. Se propuso crear un espacio para reflexionar, desde las artes visuales, acerca del lugar de las mujeres en la sociedad y, con ello, problematizar los movimientos sociales y sus discursos de inclusión.

Este proyecto tiene como contexto las manifestaciones sociales que reivindicaban los derechos civiles de las mujeres en Chile y que fueron detonados por situaciones de abuso hacia estudiantes universitarias en el 2018, lo que en este país se denominó Mayo Feminista (Zerán, 2018). A estas mujeres se unieron los establecimientos educacionales secundarios, quienes levantaron las demandas relacionadas con la apertura de liceos masculinos a la formación mixta, toma de medidas relacionadas con acoso sexual y la disposición al diálogo con relación a la diversidad sexual (Reyes-Housholder y Roque, 2019). A esto se sumó la necesidad de considerar desde las prácticas artísticas y las reflexiones en torno a las artes visuales, temáticas relacionadas con la configuración del género, en particular lo femenino, lo que se ha ido transformando en una preocupación en nuestro país. En este contexto, las prácticas artístico-culturales son una instancia no solo para construir conocimiento, sino también para llevar a cabo reflexiones significativas en torno al control y la dominación de las representaciones del cuerpo (Preciado, 2016), los afectos, el reconocimiento de autoridad femenina, las sexualidades (Witting, 1992, las visualidades que exacerban los roles y estereotipos de género (Montenegro, 2019b) y las subjetividades y configuraciones del género femenino a partir de las propias experiencias cotidianas.

En este contexto, el ejercicio docente que se desarrollaba en el Liceo n. ${ }^{\circ}$ 1 de niñas en Santiago se vio atravesado por una serie de acciones de protesta por los motivos mencionados, situaciones que fueron permeando el hacer de la escuela. Este espacio de discusión hizo que el profesorado cuestionara su rol dentro del territorio escolar y reflexionara en torno a qué y cómo se estaba enseñando. En el caso de la artista-docente que nos ocupa, ella siguió de cerca las acciones del movimiento feminista y expresó que se posicionó como "una agente activa de cuestionamiento de sistemas patriarcales de opresión, propiciando las reflexiones entre y con sus estudiantes". 
A medida que fueron avanzando las protestas y los movimientos ciudadanos, la artista-docente señaló que algunas de sus banderas de lucha se fueron transformando desde los reclamos de la población femenina en cuanto a los abusos de poder y control, hasta el reconocimiento de las diversidades sexuales. Esto último propició —en ella—, la problematización del lenguaje sexista y dio lugar a un cambio, al pasar del relato con un masculino plural a utilizar palabras que involucraban a todas las personas. Estos cambios del lenguaje, a lo largo de las movilizaciones, hicieron pensar a la artista-docente en el lugar de las mujeres.

Comenta la artista-docente a cargo del proyecto que "comenzaban las movilizaciones con reivindicaciones femeninas, sin embargo, es el propio movimiento y las nuevas propuestas lingüísticas las que volvían a invisibilizar a las mujeres". ¿Dónde quedábamos las mujeres en este relato? - se preguntaba la artista-docente- ¿Cómo nos podíamos visibilizar nuevamente dentro de esta amplitud de demandas sociales? A través de estas preguntas, la artista-docente fue buscando la forma en que la clase de Artes Visuales se transformara en un espacio reflexivo, que articulado por la práctica artística colectiva del curso, promoviera nuevas relaciones y formas de re-comprenderse. A partir de esta búsqueda, la artista educadora fue re-conceptualizando el rol de las artes dentro de la propia clase, desarrollando una experiencia en la cual la creación artística actuaba como mediadora al ser una herramienta de reflexividad visual (Montenegro, 2019b).

Con relación a esta búsqueda, en la artista-docente surge la necesidad de problematizar y reflexionar sobre el lugar de las mujeres en el contexto social y los roles que las estudiantes desempeñan. Otro tema que apuntó la artista-docente fue el debate que se produjo en el contexto estudiantil sobre los estereotipos de belleza física, los que fueron fuertemente cuestionados por las estudiantes, intentando alejarse de los estereotipos de género que se relacionan con lo femenino (pelo largo, maquillajes suaves, disposición al servicio, entre otros) (Preciado, 2016; Witting, 1992). Cuenta la artista-docente que estas temáticas que estaban presentes en su vida diaria y la del estudiantado, la impulsaron a promover que el grupo fuera dialogando sobre los procesos de aprendizaje y pudiera ir reconociendo figuras femeninas relevantes asociadas a los territorios que habitaban.

La artista-docente relata que al principio la indicación al grupo le parecía vaga y confusa, porque no existía una calificación individual asociada a la actividad, lo que les producía profunda incomodidad. ¿Mi calificación depende de mi compañera? O ¿ Tengo que ayudarla a avanzar? fueron preguntas frecuentes durante la actividad, que evidenciaban una práctica escolar ajena a la colaboración, tendiente al trabajo individual, competitivo, ausente de reflexión, puesto que normalmente seguían las instrucciones sin detenerse a cuestionarlas. 
Para la artista-docente, los objetivos del proyecto fueron reflexionar sobre el rol de las mujeres que ellas reconocían como autoridad femenina (Rivera, 2012), para que pudieran reconocer qué hay de esas mujeres que las formaron en ellas mismas y cómo construyen relación a través de los aprendizajes. Era un proyecto que invitaba a hacer genealogía en y a través de las artes (García-Huidobro, 2017), lo que transformaba al acto de mediación en un compromiso social y político y que también se transformaba en una reivindicación y reflexión de lo femenino y de los actos políticos de las mujeres.

Estos aspectos eran vitales en la presentación de la actividad puesto que, como objetivo, se perseguía que la experiencia fuera ajena a las experiencias anteriores, que no estuviera condicionada por la calificación o la necesidad de un trabajo individual. De esa manera, la docente perseguía que "la experiencia personal de fascinación por la maravilla de aprender" (Rivera, 2012, p. 89) estuviera presente en el proyecto. Con esto, para la artista-docente era importante situar la reflexión a nivel geográfico, dado que el liceo es un lugar de encuentro de estudiantes de toda la Región Metropolitana de Chile, quienes planteaban la importancia de los desplazamientos y la apropiación de los espacios en los que ellas muchas veces estaban en tránsito, como la propia escuela. Esto, la artista-docente también lo vinculó con la construcción de identidad juvenil, marcada por la marginalidad social de la escuela pública y sus barrios de origen que, en su mayoría, configuran la periferia urbana de la región.

Con estas reflexiones, la artista-docente y el estudiantado se propusieron realizar una cartografía colaborativa, que sería una reflexión visual en torno a la presencia de mujeres en sus procesos de aprendizaje. Se consideró el uso de la cartografía como una estrategia visual poscualitativa y materialista que permite acercarse a la realidad y la educación desde elementos como la reflexión, la transformación, el afecto y otras sensibilidades para promover reflexiones que formaran nuevas subjetividades (Risler y Ares, 2013). Dichas reflexiones de la cartografía se materializarían en un bordado en tela, donde cada estudiante tenía dos botones, uno donde marcaba el lugar de residencia de la mujer elegida para el proyecto, y el otro en su propio lugar de residencia.

Dada la relevancia de la acción de bordar, como un acto propiamente femenino de sus abuelas y madres, la artista-docente cuenta que se decidió utilizar esta técnica como medio para construir los saberes de aquellas mujeres que eran referentes en las historias de las estudiantes. Sobre estas ideas, tanto la profesora como el grupo eligieron en conjunto la actividad de bordar, dado que durante muchos años fue una actividad prohibida en el Liceo. Durante el siglo xx era una asignatura obligatoria, cuyo objetivo era formar mujeres capaces de hacer las ropas de sus futuras casas como esposas y madres (a esto se suma también tejer y cocinar). Es por lo anterior 
que, entrado en el siglo xxı, la actividad desapareció del currículum esco lar, que se centró en aspectos que impulsen a las estudiantes a seguir estudios superiores y dio espacio a asignaturas que las prepararan para eso. En este contexto, la artista-docente cuenta que el bordado se transformó en una acción reivindicativa de relación entre mujeres, como una acción mediadora de saberes y que en su proceso permite no solo el diálogo, sino también la valoración legítima del trabajo de otras, la narrativa oral de las vidas de las mujeres reconocidas y de los afectos que las unen a estas personas.

Así, los mapas se transformaron en la organización visual del tema tratado y se constituyeron como una reflexión visual que dio cuenta de las propias experiencias y contextos de quienes participaron en el desarrollo de la obra (Montenegro, 2019a), una experiencia desjerarquizada tanto metodológica como visualmente, a la manera de un rizoma. Guattari y Deleuze plantean también el rizoma como mapa de experiencias, donde "el mapa no reproduce un inconsciente cerrado sobre sí mismo, lo construye. Contribuye a la conexión de los campos, al desbloqueo de los cuerpos sin órganos, a su máxima apertura en un plano de consistencia" (Guattari y Deleuze, 1994, pp. 9-10). Por otro lado, se piensa que el trabajo de una cartografía colaborativa responde a las "metodologías que van siendo" (Lather, 2013, p. 365) y revela nuevos métodos artísticos que, desde las ciencias sociales, se alejan de los modos tradicionales de aprender $y$ hacer artes para problematizar aspectos que trascienden el giro reflexivo y que se vinculan con la acción, la experimentación y los afectos.

A través de estas ideas, para la artista-docente fue relevante que la actividad estuviese presente y fuera colaborativa, puesto que muchas de las reflexiones del movimiento feminista buscaban la relaciones entre mujeres de manera desinteresada, cuestionando la autoría como ejercicio de reconocimiento para pasar a una autoría colectiva. Por otra parte, se relata que fueron fundamentales en el proyecto dos aspectos. En primer lugar, el contexto y la experiencia para aprender, pues "estas ideas implican aprender en relación y poseer un sentido colaborativo de los procesos de aprendizaje" (Montenegro, 2019a, p. 292). Teniendo esto presente, la artista-docente organizó las sesiones en la Unidad 1: Problemáticas juveniles y medios contemporáneos, y se consideró el OA1: Crear proyectos visuales basados en la valoración crítica de manifestaciones estéticas referidas a problemáticas sociales y juveniles, en el espacio público y en diferentes contextos.

Se pudo observar que, si bien la actividad se desplazó del objetivo, parece relevante considerar una coyuntura social como el movimiento feminista para vincularlo con la unidad docente. La artista-docente cuenta que esta propuesta fue celebrada por la Unidad Técnico Pedagógica de la escuela, al tener en cuenta también la opinión de las estudiantes. La profesora dividió la actividad en cuatro sesiones, siendo la primera la de mayor contenido dialógico, pues, entre el grupo se expusieron las razones de la 
elección de las mujeres relevantes. La mayoría de mujeres que emergieron desde las estudiantes fueron abuelas, hermanas y madres, en su totalidad mujeres con un vínculo sanguíneo; a través de los relatos orales se reconoció una relación de cuidado y amor que permitió identificar los procesos de mediación del aprendizaje desde la genealogía (Flecha, 2006). Las dos sesiones siguientes fueron un trabajo de bordado y pegado de botones (véanse las fotografías 1 y 2), para terminar con una sesión de presentación de la cartografía y su montaje en un espacio público de la escuela. La actividad no solo abordó un proceso artístico colaborativo, sino que también permitió relevar un hacer artístico que valora el tiempo de dedicación, el acto del encuentro entre compañeras mujeres y el ejercicio dialógico constante a lo largo del trabajo de bordado. Esto contribuyó a llevar a cabo procesos de reconocimiento de subjetividades donde los afectos cobran un lugar central, lo cual a su vez les permitió analizar el contexto escolar que se desplazó de estos últimos, valorando la instancia de cuestionamiento a la que invita la actividad y la asignatura.

Otro aspecto importante que se observó en esta actividad fue la reflexión en torno a cómo las estudiantes reconocían sus propias trayectorias como mujeres. Desde un acto colectivo de bordado, esto abre la puerta a pensar en el reconocimiento femenino desde las propias subjetividades, pero también como un acto político de reivindicación de labores femeninas. Así, las estudiantes en medio de las movilizaciones feministas ven en la actividad una forma de resistencia a un sistema social que prioriza la rapidez y la producción en serie; así, el bordado se transforma en un espacio de protesta y de valoración del cuidado, el diálogo entre mujeres y el reconocimiento de las genealogías femeninas.
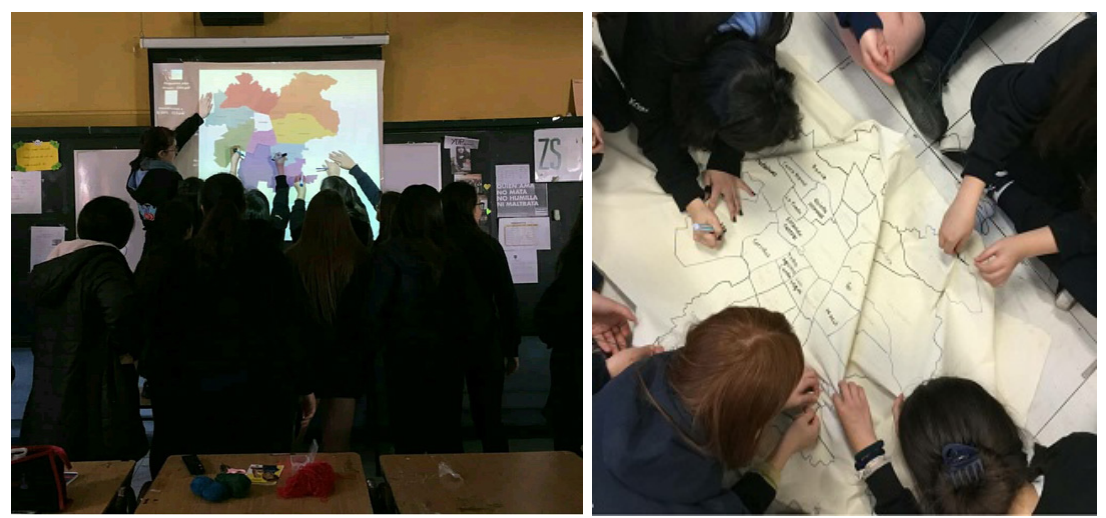

Fotografias 1 y 2. Diseño del mapa de la Región Metropolitana en el proyecto "La cartografía de los aprendizajes amorosos: reconocimiento de autoridad femenina en los procesos de aprendizaje" (2018) 
Cuenta la artista-docente que, durante el proceso, las estudiantes dividieron las tareas. Así, mientras unas dibujaban el mapa en la tela (fotografía 1), otras se encargaban de ir organizando los hilos para comenzar a bordar (fotografía 2). El trabajo colaborativo tuvo entonces un lugar relevante como experiencia de sentido y como ejercicio de mediación, puesto que, como señala Brown (2017), el cuestionamiento sobre las formas de hacer en sociedad y las creaciones y prácticas artísticas de protesta (enmarcadas en contextos particulares) invitan al diálogo y a la problematización de la propia creación artística. Así, en palabras de Cortés, es posible visibilizar a través de las prácticas artísticas femeninas "un relato que considere el esquema de la subjetividad y la circulación de cuerpo, del discurso y del territorio político implícitos en sus procesos creativos y de difusión de obra" (2017, p. 15). De esta manera, el proyecto es mediación en la medida en que dialoga entre lo artístico y pedagógico, como una acción propiciadora de diálogo crítico y cambio social, hacia una conciencia del ser situado en femenino (Montenegro, 2019c).

También, se puede analizar que este fue un proyecto que puso en juego las mediaciones entre el grupo de estudiantes — como aquello que Lia Cigarini (2009) denomina la política de las relaciones - y se vincula con los saberes que se van conformando a través de las vinculaciones y las mediaciones con otras mujeres. Frente a ello, valoramos y reconocemos cómo este proyecto se va articulando como un compromiso social que incluye a la otras como propia medida, para reconocerse y reconocerlas.

Esta actividad nos permite comprender la práctica artística cartográfica de este proyecto, cercana al activismo y las reflexiones colectivas en torno a la condición femenina. Según lo anterior, es posible identificar algunos elementos que transitan desde y hacia las artes como espacios formativos y de la sociedad general, como el respeto, la colaboración y la equidad (Huerta, 2016). Según lo anterior, en este proyecto el arte sería no solo un acto mediador-transformativo, sino también un espacio revitalizante (Bourriaud, 2009) y posibilitador de nuevas subjetividades femeninas en el territorio escolar. Es en este contexto que se da valor a la colectividad, las propias historias de vida, las experiencias y los contextos (Montenegro, 2018) del hacer artístico-cultural, lo que concuerda con los planteamientos del arte comunitario en sus inicios en las décadas de los 60 y 70 en Inglaterra, donde se enfatiza el proceso de aprendizaje colectivo (Birchall, 2017). Esta experiencia artística pedagógica se podría plantear como "espacios de devenir o agenciamiento, en los que el trabajo es producido de forma transversal, rompiendo las jerarquías impuestas por la modernidad y abogando por una continua apropiación y dispersión de la cultura" (Blanco, 2001, p. 15). Según esto, es posible visualizar un proyecto que concibe la práctica artística como agente articulador de subjetividades en clave femenina. 

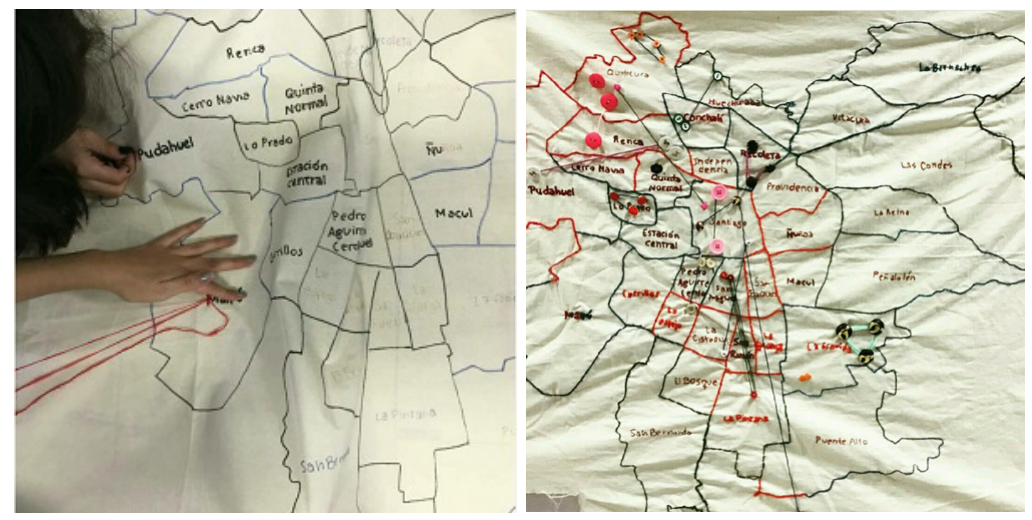

Fotografias 2 y 3 . Comienzo del proceso y resultados de bordado en el proyecto "La cartografía de los aprendizajes amorosos: reconocimiento de autoridad femenina en los procesos de aprendizaje" (2018) Fotografia de Catalina Montenegro

Por otro lado, la artista-docente señala que el trabajo realizado con las estudiantes le permitió reflexionar sobre su rol dentro de los procesos creativos en el aula. Al respecto, expresó:

mi experiencia docente y la relación con la creación artística va más allá de la clase de la escuela y se puede pensar como una mediación con el mundo cultural y la búsqueda de otras mediaciones que pudieran hacer pervivir esta relación más allá de la clase y transitar definitivamente hacia la mediación desde lo pedagógico, trascendiendo el territorio escolar.

Consideramos relevante la reflexión de la artista educadora, puesto que

La educación es el lugar donde la rareza y el imprevisto se dan la mano; el lugar de las curiosidades compartidas, las subjetividades compartidas, los sufrimientos compartidos, las pasiones compartidas que se congregan hacia la promesa de un tema, de un conocimiento, de una posibilidad creativa. (Rogoff, 2008)

Tomando las palabras de Rogoff, el proyecto relatado plantea esa anomalía, esa grieta en el territorio escolar que trasciende la propia escuela y se apropia de los discursos sociales que se comparten en el aula para llevar a cabo procesos creativos en la colectividad femenina.

Además, este tipo de propuestas artísticas y en colectivo muestra nuevas formas en las que se posiciona los y las artistas o artistas-docentes, quienes se colocan como sujetos que posibilitan reflexiones o preguntas $y$, además, aprenden desde esos espacios. Esto enseña la importancia de las relaciones en la enseñanza de las artes (Porres, 2012), como un elemento de mediación, donde el pensar y hacer artístico promueven subjetividades que se piensan colectivamente. 


\section{Conclusiones}

Este artículo, que se sitúa en el marco de una investigación sobre el cruce artístico y pedagógico de artistas-docentes, nos plantea aspectos relevantes sobre las nuevas formas de pensar las artes y su producción, unas que van más allá del arte propositivo, de los espacios artísticos tradicionales y los círculos que avalan y validan este campo. La emergencia de nuevas propuestas artísticas ligadas a contextos sociales plantea otras formas de entender las artes con un sentido político y transformador (Guerra, 2017).

El proyecto artístico "La cartografía de los aprendizajes amorosos: reconocimiento de autoridad femenina en los procesos de aprendizaje" plantea la riqueza de trabajar reflexiones visuales situadas en un contexto escolar público, donde emergen elementos relacionales y afectivos y en donde lo artístico se transforma en un vehículo mediador de nuevas realidades. A través de estas propuestas, se potencia el pensamiento crítico de las estudiantes y las artes propician el diálogo de las propias experiencias y las relaciones de aprendizaje en femenino.

Tras esta experiencia, pensamos que los proyectos de trabajo artísticos con el estudiantado permiten dar un giro desde la creación artística como respuesta a las necesidades de la escuela a un espacio de reflexión crítica a partir de coyunturas sociales, es decir, son las propias dinámicas sociales las que pueden articular proyectos en los contextos escolares, yendo más allá de los requerimientos de las asignaturas para lograr experiencias significativas de mediación, tanto para el estudiantado como para las y los docentes a cargo.

En este sentido, existe una re-significación de la creación artística, que en un contexto escolar se activa como mediación social y como una práctica dialógica que puede propiciar múltiples iniciativas a partir de una mirada de la escuela a la sociedad y los territorios en los que se encuentra inmersa. Según esto, los aportes que puede presentar un proyecto con estas características a las artes visuales y a la educación artística se relacionan con lo mencionado: un proceso de reflexión basado en los procesos creativos visuales que permiten tratar temas de contingencia a través de una práctica artística colaborativa, que además puede poner en valor actividades propias de la historia cotidiana y del mundo privado del estudiantado que, en el caso de este proyecto, se reconocen en el bordado. Esto permite problematizar la actividad de bordar para las estudiantes, puesto que no es parte de la cotidianeidad de las jóvenes y les permite resignificar el acto del trabajo hecho a mano, alejadas de la inmediatez, el individualismo y la competitividad que ellas mismas manifiestan como presentes en sus rutinas escolares. 
A través de la reflexión del proyecto "La cartografía de los aprendizajes amorosos: reconocimiento de autoridad femenina en los procesos de aprendizaje", podemos señalar que los proyectos que se sitúan desde la mediación permiten reflexionar sobre creaciones artísticas que se alejan de la autoría como eje de las propuestas artísticas, pues al situar el foco en el proceso de mediación se visibilizan los aspectos más políticos y transformativos de las artes (García-Huidobro, 2019). Estos son los que cruzan el sentido personal y social, uno que siempre es ideológico, porque se arraiga en una experiencia mediada por el cuerpo y la alteridad y que se mueve entre nuestras creencias y deseos de quienes viven la experiencia artística. Además, se considera que los proyectos artísticos pedagógicos que se conforman desde la mediación poseen un compromiso político con la práctica social que nos lleva a entenderlos como transformativos.

En este proyecto se buscó generar nuevos significados, maneras de comprender a las mujeres estudiantes y sus subjetividades. Este compromiso político con lo personal, como mujeres, busca la transformación del orden social y nos enseña "la idea de libertad relacional en el arte, porque la experiencia artística es vivida con y como libertad femenina política" (García-Huidobro, 2017, p. 607).

Finalmente, diremos que el acento de los proyectos artísticos y educativos que surgen desde la mediación y la promueven no están centrados en el objeto o resultado artístico, sino en el proceso dialógico y posibilitador de subjetividades. Este tipo de propuestas suscita un sentido trascendente, que va más allá del objeto y pone el foco en la experiencia artística que se vive y comparte, donde se puede ocupar un papel metodológico, de mediación o transformación, que lleva a un grupo a aprender para transformar la realidad social. La mediación es un acto de aprendizaje en el cual las relaciones quedan en evidencia en el proceso de creación artística y por ello es importante documentar dicho proyecto. La mediación es, a su vez, una forma de compartir y aprender saberes, donde lo artístico se convierte en posibilitador y activador de la experiencia, un espacio donde emerge lo que Hernández (2011) ha llamado encuentro de subjetividades.

\section{Referencias}

Barbosa, A. (2002). Arte, educación y reconstrucción social. Cuaderno de Pedagogía, 311, 56-58.

Birchall, M. (2017). Situating participatory art between process and practice. Arken Bulletin, 7, 56-74.

Blanco, P. (2001). Explorando el terreno. En P. Blanco (ed.), Modos de hacer: Arte crítico, esfera pública y acción directa. Ediciones Universidad de Salamanca. 
Bourriaud, N. (2009). Postproducción. La cultura como escenario: Modos en que el arte reprograma el mundo contemporáneo. Los Sentidos/ Artes Visuales.

Brown, W. K. (s. f.). Questions for social practice art. https://www.academia.edu/6103828/Questions_for_Social_Practice_Art

Calderón, N. y Ortiz, J. (2019). Practicar la inestabilidad: Diálogos y acercamientos desde la investigación artística. Universidad Veracruzana.

Carrasco, S. V. y García-Huidobro, R. (2017). Repensando nuestros posicionamientos como profesoras de arte dentro de un escenario de cambios. Educación Artística: Revista de Investigación (EARI), 8, 58-73.

Cercós y Raichs, R. (2017). El pensamiento estético-pedagógico de Joseph Beuys: Entre la memoria y la performance. Historia y Memoria de la Educación, 5, 217-237. https://doi.org/10.5944/hme.5.2017.16797

Chase, S. (2011). Narrative inquiry: Still a field in the making. En Denzin y Lincoln (eds.), The Sage handbook of qualitative research (pp. 421434). Sage.

Cigarini, L. (2009). Coloquio con Lia Cigarini, "La pasión por la política: pensar las relaciones y el trabajo" (4 Diálogo Magistral del Centro de Investigación Duoda). Duoda: estudis de la diferència sexual, 37, 89-99.

Coca, P. (2011). Procesos de mediación en las prácticas comisariales. EARI Educación Artística. Revista de Investigación, 2, 71-75.

Cortés, Gloria. (2017). Desacatos: Prácticas artísticas femeninas (18351938). Colección mNBA. Dibam.

De Pascual, A. y Lanau, D. (2018). El arte es una forma de hacer (no una cosa que se hace). Catarata.

Duchamp, M. (1999). The creative act. Sub Rosa.

Flecha, C. (2006). Genealogía. En A. M. Piussi y A. Mañeru (ed.), Educación nombre común femenino (pp. 46-65). Octaedro.

Fontdevila, O. (2018). El arte como mediación. http://oriolfontdevila.net/ wp-content/uploads/2018/04/Art-mediation_Homesession-2017_ ENG.pdf

García-Huidobro, R. (2017). Retratar a otras mujeres y cartografiar nuestros vínculos. Investigaciones Feministas, 8(2), 603-619. https://doi. org/10.5209/INFE.54143

García-Huidobro, R. (2018a). Artistas-docentes que aprenden a enseñar: Abrir espacios pedagógicos y transgredir dualidades. Innovación Educativa, 18(77), 39-56.

García-Huidobro, R. (2018b). El lugar del conflicto en la experiencia de saber pedagógico de profesoras de artes en educación secundaria. Revista Complutense de Educación, 29(4), 959-973. https://doi. org/10.5209/RCED.54399 
García-Huidobro, R. (2019). Prácticas y producciones artísticas que se re-significan desde lo pedagógico. Revista Estudios sobre Arte Actual, 7, 211-217.

García-Huidobro, R. (s. f.). El método de taller artístico de discusión para investigar la experiencia de artistas-docentes en la era postcualitativa. Empiria. En proceso de revisión de pares.

García-Huidobro, R. (2020). Cruzar la mirada: Resignificar a las artes en la sociedad actual. Ril Editores.

Guattari, F. y Deleuze, G. (1994). Rizoma (Introducción). Diálogo Abierto.

Guerra, L. (2017). De la inexistencia del arte. Brumaria.

Hall, J. (2010). Making art, teaching art, learning art: Exploring the concept of the artist teacher. International Journal of Art \& Design Education, 29(2), 103-110. https://doi.org/10.1111/j.1476-8070.2010.01636.x

Hernández, F. (coord.). (2011). Pensar la relación pedagógica en la universidad desde el encuentro entre sujetos, deseos y saberes. Universitat de Barcelona. Dipòsit Digital.

Hormazábal González, V. (2013). La obra visual de Violeta Parra: Un acercamiento a sus innovaciones conceptuales y visuales a través del análisis iconográfico de arpilleras y óleos. [Tesis de pregrado, Universidad de Chile]. Repositorio institucional UChile http://repositorio.uchile.cl/ handle/2250/113847.

Huerta, R. (2016). Transeducar: Arte, docencia y derechos LGTB. Egales.

Lather, P. (2013). Metodology-21: What do we do in the afterward? Revista Internacional de Estudios Cualitativos en Educación, 26(6), 634-645. http://dx.doi.org/10.1080/09518398.2013.788753

Lucke, C. (1999). Feminismos y pedagogías en la vida cotidiana. Morata.

Montenegro, C. (2019a). Propuesta de didáctica feminista para la enseñanza escolar de las artes visuales: Pensar el espacio escolar de otra manera. Estudios Pedagógicos, 44(3), 289-302. http://dx.doi.org/10.4067/ S0718-07052018000300289

Montenegro, C. (2019b). Cartografías colectivas como espacios de reflexión visual: Territorios, experiencias y contextos. Acta del IV Congreso Internacional de investigación en Artes Visuales, ANIAV 2019 (pp. 1-6). Universitat Politècnica, València, España.

Montenegro, C. (2019c). La creación artística como proceso reflexivo del SER en femenino: El caso de la escuela chilena. Revista Estudios Sobre Arte Actual, 7, 213-218.

Moreno, A. (2016). La mediación artística: Artes para la transformación social, la inclusión social y el desarrollo comunitario. Octaedro.

Ossa, C. (2017). Políticas y didácticas. Estudio sobre educación artística: Creatividad social ciudadana en el CNCA. Universidad de Chile. 
Porres, A. (2012). Relaciones pedagógicas en torno a la cultura visual de los jóvenes. Octaedro.

Preciado, P. (2016) Manifiesto contrasexual. Anagrama.

Ramos, D. (Ed.). (2019). Miradas caleidoscópicas: Educación artística visual en las culturas contemporáneas. Universidad Pedagógica Nacional.

Reyes-Housholder, C. y Roque, B. (2019). Chile 2018: Desafíos al poder de género desde la calle hasta la Moneda. Revista de Ciencia Política, 39(2), 191-215.

Risler, J. y Ares, P. (2013). Manual de mapeo colectivo: Recursos cartográficos críticos para procesos territoriales de creación colaborativa. Journal of Latin American Geography, 16(2), 154-156.

Rivera, M. (2012). El amor es el signo: Educar como educan las madres. Sabina.

Rodrigo, J. y Collados, A. (2015). Retos y complejidades de las prácticas artísticas colaborativas y las pedagogías colectivas. Pulso, 38, 57-72.

Rogoff, I. (2008). Turning. E-flux, 0. https://www.e-flux.com/journal/00/68470/ turning/

Rogoff, I. (2011). El giro. Arte y políticas de identidad, 4, 253-266.

Wilde, O. (1898). La decadencia de la mentira. Taurus.

Wittig, M. (1992). El pensamiento heterosexual y otros ensayos. Reacia Ediciones.

Zerán, F. (Ed.). (2018). Mayo feminista: La rebelión contra el patriarcado. LOM.

Zúñiga, D. (2019). Los sentidos pedagógicos en una práctica artística dirigida para jóvenes dentro de un espacio cultural en Chile [tesis de doctorado]. Universidad de Barcelona. 